\title{
IMF-By dependence of transient ionospheric flow perturbation associated with sudden impulses: SuperDARN observations
}

\author{
Tomoaki Hori ${ }^{1 *}$, Atsuki Shinbori², Shigeru Fujita ${ }^{3}$ and Nozomu Nishitani ${ }^{1}$
}

\begin{abstract}
A statistical study using a large dataset of Super Dual Auroral Radar Network (SuperDARN) observations is conducted for transient ionospheric plasma flows associated with sudden impulses (SI) recorded on ground magnetic field. The global structure of twin vortex-like ionospheric flows is found to be consistent with the twin vortices of ionospheric Hall current deduced by the past geomagnetic field observations. An interesting feature, which is focused on in this study, is that the flow structures show a dawn-dusk asymmetry depending on the combination of the polarity of SI and interplanetary magnetic field (IMF)-By. Detailed statistics of the SuperDARN observations reveal that the dawn-dusk asymmetry of flow vortices due to IMF-By appears during negative Sls, while such asymmetric characteristics are not seen during positive SIs. On the basis of the upstream observations, we suggest that this particular dawn-dusk asymmetry is caused by the interaction between the pre-existing round convection cell and a pair of the transient convection vortices associated with SIs.
\end{abstract}

Keywords: Sudden impulse, lonospheric plasma convection, SuperDARN

\section{Introduction}

Sudden impulse (SI) is a rapid increase or decrease in intensity of a geomagnetic field recorded almost simultaneously by the world-wide network of magnetic observatories and typically lasts for a few tens of minutes (Araki 1977). Such geomagnetic variations are caused mainly by interplanetary shocks and tangential discontinuities propagating in the solar wind (Chao and Lepping 1974). Both stepwise increases and decreases of the geomagnetic field are often observed globally (Nishida and Jacobs 1962a; Nishida and Jacobs 1962b) and are referred to as positive sudden impulses (positive SIs) and negative sudden impulses (negative SIs), respectively. They are thought to correspond to global compressions or expansions of the magnetosphere induced by abrupt changes in the solar wind dynamic pressure $\left(P_{\mathrm{SW}}\right)$ (Wilken et al. 1982; Tsurutani et al. 1995).

\footnotetext{
* Correspondence: tomo.hori@nagoya-u.jp

${ }^{1}$ Institute for Space-Earth Environmental Research, Nagoya University,

Furo-cho, Chikusa, Nagoya, Aichi 464-8601, Japan

Full list of author information is available at the end of the article
}

The global characteristics of ground magnetograms have been extensively investigated by the past studies. Araki (1994) developed a physics-based model of the disturbance field associated with an SI based on a synthesis of the extensive observational results from ground magnetometers and the theoretical consideration for propagation of hydromagnetic waves in the magnetosphere-ionospherecoupled system (Tamao 1964a; Tamao 1964b). One of the important aspects of this model is that geomagnetic disturbances associated with an SI consist of two basic components appearing primarily at low latitudes (DL) and in the polar region (DP). The former component is attributed to contributions from variations in the Chapman-Ferraro current along the magnetopause, while the latter comes mainly from the twin vortex Hall current induced in the ionosphere. In addition to the spatial structure, a two-phase perturbation with a preliminary impulse (PI) and the subsequent main part of an SI, referred to as main impulse (MI), are interpreted as an alternate evolution of the twin vortex current systems of opposite polarity.

\section{实 Springer}


Besides the geomagnetic field observations, the Super Dual Auroral Radar Network (SuperDARN) (Greenwald et al. 1995) has played an important role in the measurement of the two-dimensional structure of ionospheric convection associated with SIs (Lyatsky et al. 1999; Thorolfsson et al. 2001; Vontrat-Reberac et al. 2002; Coco et al. 2008; Huang et al. 2008; Kane and Makarevich 2010; Liu et al. 2011; Gillies et al. 2012; Hori et al. 2012). A conclusion from these studies with the radar observations is that changes in flow direction and the polarity of flow shear are consistent with a PI-MI sequence on ground magnetograms described by the SC model proposed by Araki $(1977,1994)$. The same characteristics have been confirmed further by examining not only simple flow directions but also the polarity of ionospheric flow shear corresponding to the dusk cell of the twin vortex current system (Liu et al. 2011; Hori et al. 2012).

Although the radar observations give flow patterns consistent with the ground magnetic field perturbation, their observations in the past studies have been performed for spatially limited areas due to limited number of radars available for their analyses. Recent growth of the radar network (Lester 2014), however, paves the way to study the large-scale profile of SI-induced ionospheric flow perturbation solely based on the SuperDARN measurement. A great advantage of using the radar observation is that observed flow velocities are not biased by the non-uniformity of ionospheric conductance. The purpose of the present study is to examine global structure of ionospheric flow perturbation associated with SIs by using SuperDARN observations. In particular, we focus on dependence of SI-induced flows on the By component of interplanetary magnetic field (IMF), which has not been fully investigated by the past studies using geomagnetic field observations.

\section{Data and method}

In the present study, we statistically analyze horizontal ionospheric flows observed by SuperDARN radars during SI periods. First, potential SI events were picked up by identifying particular SYM-H variations with the following criteria: (1) $|\Delta \mathrm{SYM}-\mathrm{H}|>5 \mathrm{nT}$; (2) $|\mathrm{dSYM}-\mathrm{H} / \mathrm{dt}|>$ $0.025 \mathrm{nT} / \mathrm{s}(\sim 15 \mathrm{nT} / 10 \mathrm{~s})$; (3) $|\mathrm{dSYM}-\mathrm{H} / \mathrm{dt}|<0.015 \mathrm{nT} / \mathrm{s}$ during preceding $40 \mathrm{~min}$; and (4) SYM-H $>-40 \mathrm{nT}$, $\mathrm{AE}<200 \mathrm{nT}$. As done in the previous studies (e.g., Shinbori et al. 2012), we used the SYM-H index to identify SI because its nature of averaged low-latitude geomagnetic variations is suitable to capture rapid changes of the magnetopause current causing a SI. Criteria (1), (2), and (3) were set to identify well-defined SI events with significant amplitude and rapid change in SYM-H following fairly steady periods. Criterion (4) was set to limit the present analysis to clear SI events during geomagnetically quiet periods not to mistakenly pick up random, highly variable periods of SYM-H during disturbed conditions as SI events. For all of them, we visually inspected the solar wind data obtained by the ACE spacecraft to check if each of the potential SI events was associated with a rapid rise or drop of the solar wind dynamic pressure $\left(P_{\mathrm{SW}}\right)$, considering the solar wind transit time from the ACE location to the Earth. This selection procedure results in 192 positive SI events and 179 negative SI events during March 2007-August 2014. For each SI event, we define the SI onset time $\left(t_{\mathrm{s}}\right)$ and rise time $\left(T_{\text {rise }}\right)$ as schematically shown in Fig. 1. Further, the SI peak interval $\left(T_{\text {peak-SI }}\right)$ and the pre-SI interval $\left(T_{\text {pre-SI }}\right)$ are defined to be time periods of $\left[t_{\mathrm{s}}+2 \times T_{\text {rise }} / 3, t_{\mathrm{s}}+4 \times T_{\text {rise }} / 3\right]$ and $\left[\mathrm{t}_{\mathrm{s}}-10 \mathrm{~min}, \mathrm{t}_{\mathrm{s}}-4 \mathrm{~min}\right]$, respectively.

All SuperDARN radar data available in the northern hemisphere are collected for the selected SI events. To examine ionospheric flow perturbation associated with SIs, we use only ionospheric backscatter data by excluding the ground scatter data with empirical criteria proposed by Sundeen et al. (2004). We calculated the average line-of-sight Doppler velocity (LOSV) at each location in the field of view of each radar, over $T_{\text {pre-SI }}$ and $T_{\text {peak-SI }}$, respectively, for each SI event. The obtained average LOSV values are sorted into 3 degrees in magnetic latitude (MLAT) $\times 2 \mathrm{~h}$ in magnetic local time (MLT) bins in the Altitude-Adjusted Corrected Geomagnetic (AACGM) coordinates (Baker and Wing 1989), as shown by red meshes in the upper panel of Fig. 2 .

The beam-swinging technique (Makarevich and Dyson 2008) is applied for each bin to deduce a twodimensional flow vector. This method has originally been used by Ruohoniemi and Greenwald (1996) (see Figure 1 in their paper) to statistically deduce the average high-latitude convection map from SuperDARN LOSV observations. Here, let us briefly explain how this method works. If ideally all LOSV measurements made for different beam directions scanned by various radars in a MLAT-MLT bin were their line-of-sight components of a single true flow vector, the LOSV value should be zero when the beam direction is exactly perpendicular to the true flow vector and the sign of LOSV flips across such an angle normal to the true flow. That is,

$$
V_{\mathrm{LOS}}=V_{\text {true }} \cos \left(\theta_{\mathrm{bm}}-\theta_{\text {true }}\right)
$$

where $V_{\mathrm{LOS}}$ and $\theta_{\mathrm{bm}}$ are the LOSV value and beam direction, respectively, and $V_{\text {true }}$ and $\theta_{\text {true }}$ are the flow magnitude and direction of the true flow velocity, respectively. Although the true flow vector varies from event to event in reality, the average true flow angle can be determined statistically by searching for $\theta_{\mathrm{bm}}$ giving a zero LOSV.

Particularly in the present study, the residual LOSV values (dLOSV) deduced by subtracting an average 


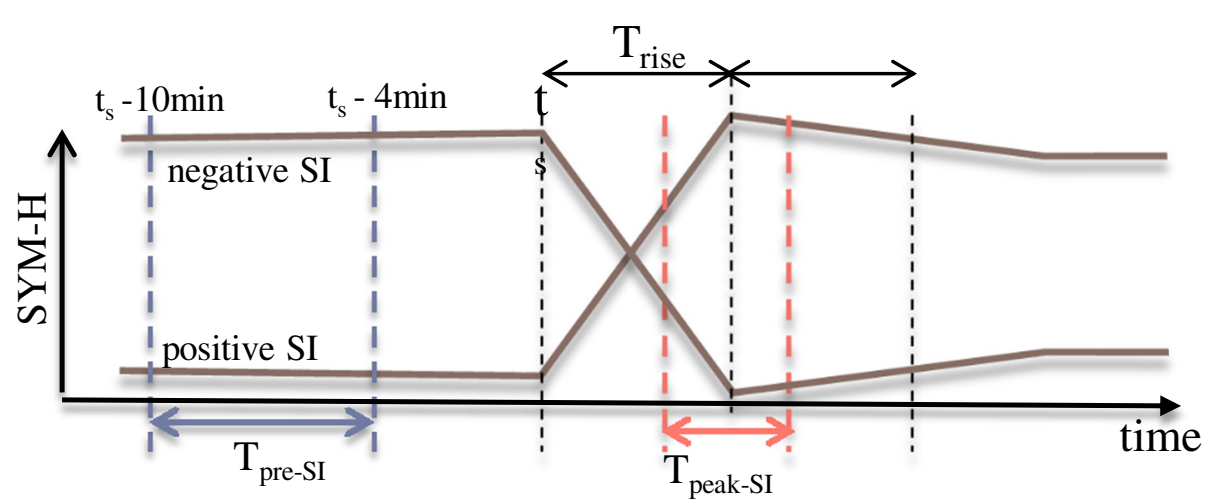

Fig. 1 A schematic of the definition of SI onset time, rise time, and SI peak period and pre-SI period

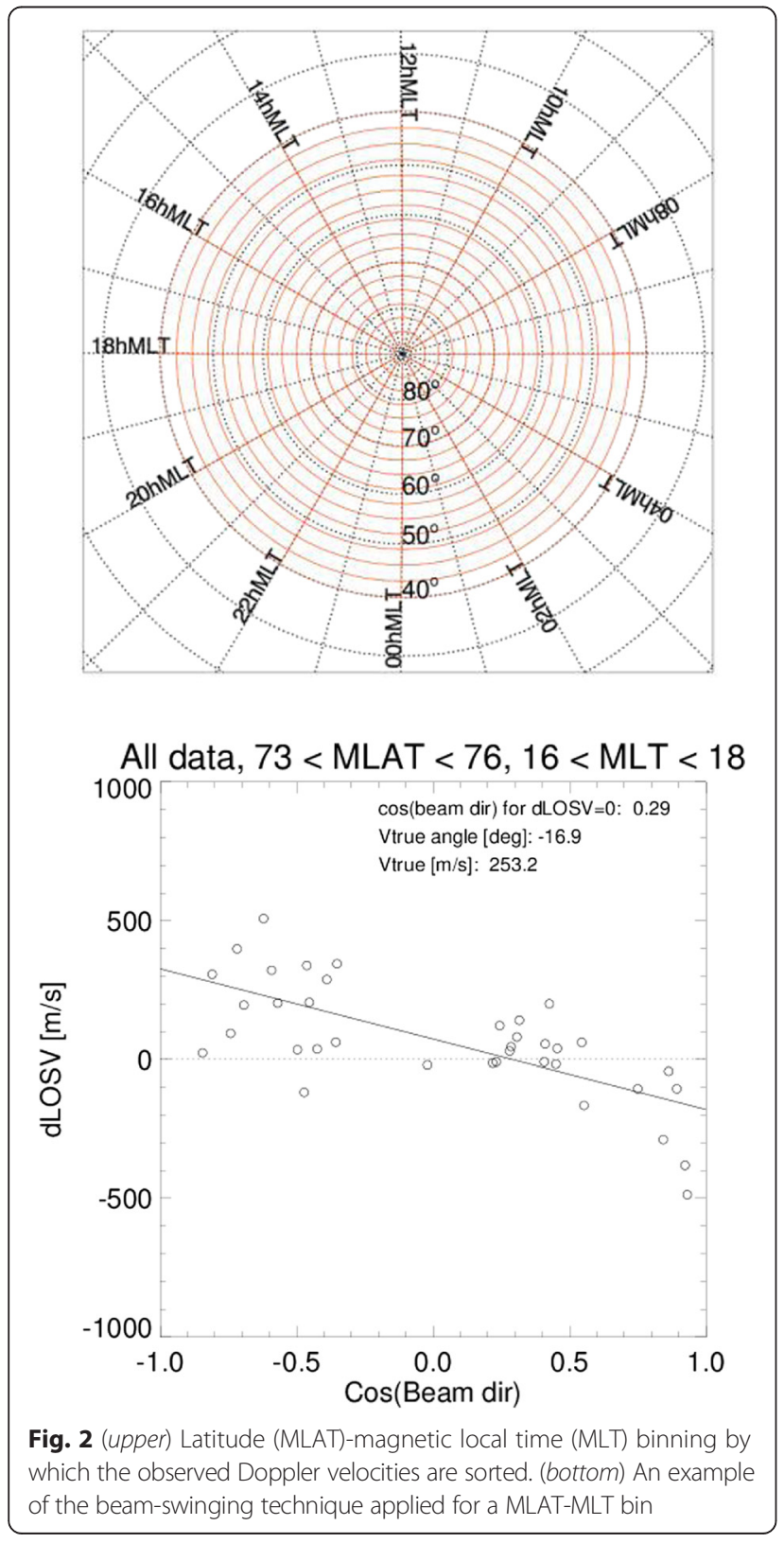

LOSV before SI from that during SI for each bin are put in the above statistical procedure to obtain a flow perturbation vector, because the flow change upon SI is to be examined. Here, all dLOSV vectors for each bin are plotted separately on a dLOSV -cosine of the beam direction plane, and the intercept of the $\cos \left(\theta_{\mathrm{bm}}\right)$ axis of a linear fitting line for the data points gives you the normal direction of a true two-dimensional flow perturbation vector and readily $\theta_{\text {true }}$ by rotating by $90^{\circ}$. The magnitude of the flow perturbation vector is also obtained as a dLOSV value at $\theta_{\mathrm{bm}}=\theta_{\text {true }}$ along the fitting line. For example, the bottom panel of Fig. 2 demonstrates the fitting result for the bin of $73^{\circ}<$ MLAT $<76^{\circ}$ and $16 \mathrm{~h}<\mathrm{MLT}<18 \mathrm{~h}$ of positive SIs. The resultant $\theta_{\text {true }}$ is $-16.9^{\circ}$, and flow perturbation magnitude is $253.2 \mathrm{~m} / \mathrm{s}$. Here, the notation of $\theta_{\text {true }}$ is the angle in the anticlockwise direction from the longitudinally eastward vector. Thus, the resultant flow perturbation is $253.2 \mathrm{~m} / \mathrm{s}$ and points roughly eastward with a small equatorward component. We applied these procedures for every bin and deduced the average pattern of flow perturbation induced by SIs.

\section{Results}

The flow perturbation associated with positive SIs and negative SIs is deduced as described in the previous section and shown in the top two panels and bottom two panels, respectively, of Fig. 3. In addition to the SI polarity, we have divided the dataset into the positive IMF-By (left panels) and negative IMF-By cases (right panels) to examine dependence of the flow perturbation on the IMF-By polarity. Here, the positive and negative IMF-By cases are based on particular SI events during which average IMF-By values for $30 \mathrm{~min}$ before and after a jump in solar wind dynamic pressure are either positive or negative. In Fig. 3, white arrows are the fitted twodimensional flow vectors, and the color of each bin represents their flow magnitude. There are two types of colors for the bins: green-blue and red-yellow mean that 


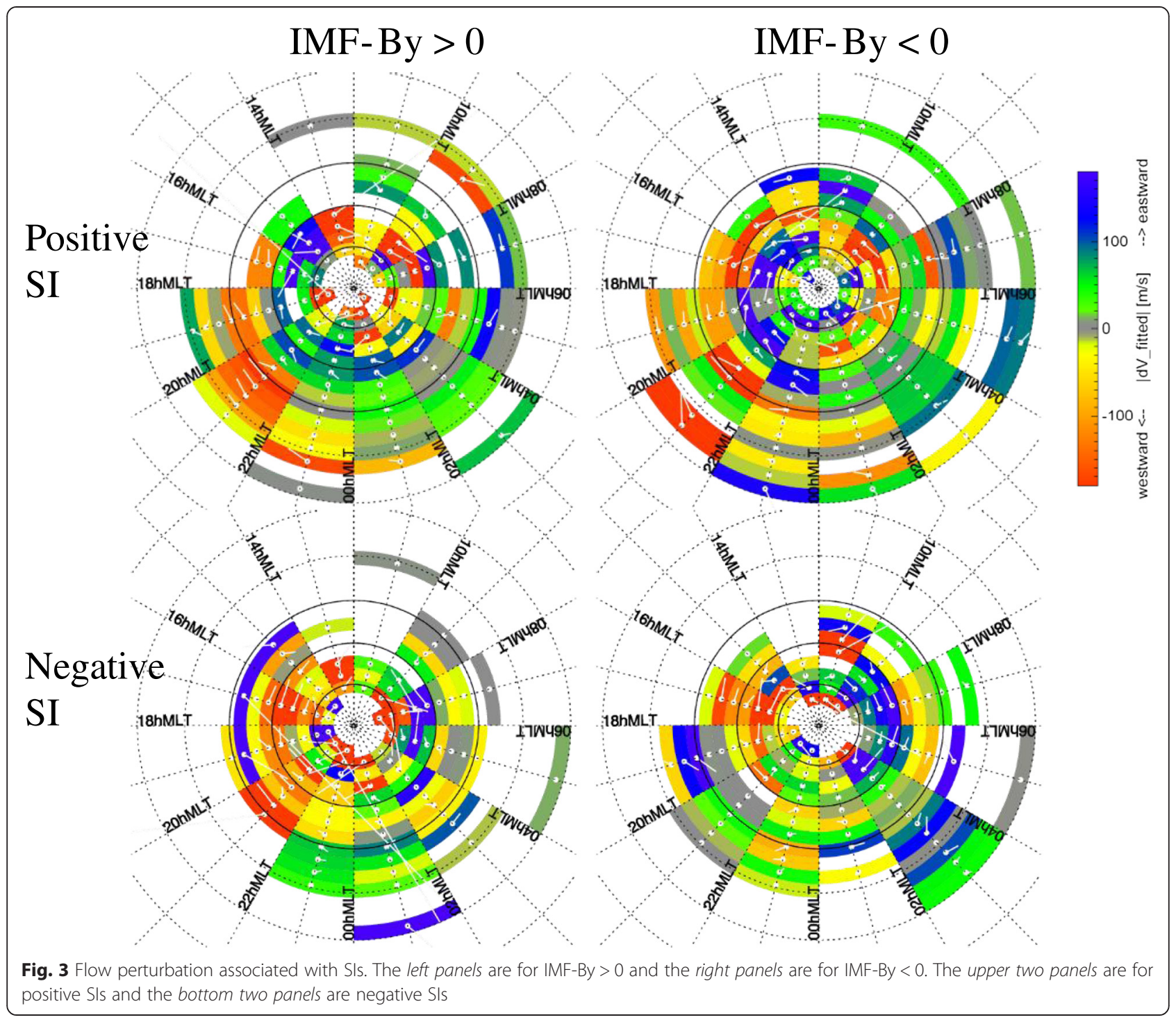

fitted velocities have an eastward or westward component, respectively.

It is seen in the top two panels of Fig. 3 that the higher latitude (roughly MLAT $>\sim 70^{\circ}-73^{\circ}$ depending on MLT) flows go anti-sunward, and the lower-latitude flows go sunward for positive SIs, roughly forming twin flow vortices with the same polarity as nominal global convection. On the contrary, the polarity of twin flow vortices is opposite for negative SIs with a boundary of sunward and anti-sunward flows at MLAT $\sim 60^{\circ}-70^{\circ}$ depending on MLT as seen in the bottom two panels. The lower latitude, anti-sunward flow is mostly weak and can barely be identified in a small number of bins, such as MLAT $<70^{\circ}$ and $4 \mathrm{~h}<\mathrm{MLT}<8 \mathrm{~h}$ and MLAT $<61^{\circ}$ and $18 \mathrm{~h}<\mathrm{MLT}<20 \mathrm{~h}$. These polarities of flow vortices are basically consistent with the Hall current vortices of MI phase associated with a positive SI and negative SI (Araki and Nagano 1988; Araki 1994; Vichare et al. 2014).
An interesting result from Fig. 3 is that the profile of higher-latitude sunward flows for negative SIs is significantly different between positive and negative IMF-By. For example, as seen from the bins of $\sim 70^{\circ}<$ MLAT $<80^{\circ}$ and $2 \mathrm{~h}<\mathrm{MLT}<10 \mathrm{~h}$ on the dawn side, the sunward flow is stronger on average and identified in more bins for IMF$\mathrm{By}<0$ than for IMF-By $>0$. On the other hand, the dusk side sunward flow is distributed in a much broader area $\left(64^{\circ}<\right.$ MLAT $<80^{\circ}$ and $14 \mathrm{~h}<$ MLT $\left.<22 \mathrm{~h}\right)$ for IMF-By $>0$ as compared with the same area for IMF-By $<0$. As a result, the sunward flow during negative SIs shows a dawndusk asymmetry in flow intensity and spatial distribution with the side of more intense sunward flow reversed depending on the IMF-By polarity. In contrast, such a tendency is hardly seen for positive SIs. Although the spatial profile of higher-latitude anti-sunward flow is more or less complicated, the relative intensity of anti-sunward flow between dawn and dusk does not seem to change much 
with the IMF-By polarity. Therefore, to explain the exclusive occurrence of IMF-By-dependent dawn-dusk asymmetry under negative SIs, there must be some mechanisms causing this that are activated only during negative SIs. We discuss some possible scenarios for it in the following section.

\section{Discussion}

The present statistics of SuperDARN data have shown that the ionospheric flow perturbation associated with positive SIs does not show a significant difference between positive and negative IMF-By polarities, while that with negative SIs has a clear dawn-dusk asymmetry, depending on IMF-By polarity. To our best knowledge, the present study is the first ever report showing such IMFBy dependence of SI-associated large-scale flow perturbation in the polar ionosphere.

There may be two possibilities causing this flow asymmetry. One is that a pair of flow vortices in association with negative SIs (Fujita et al. 2004; Fujita et al. 2012) somehow has a dawn-dusk asymmetry in flow intensity. Another possibility would be that the pre-existing round cell induced by the interaction between IMF-By and the magnetosphere (e.g., Weimer 1995; Tanaka 1999) changes its flow magnitude after SI onset. Let us discuss them in more detail below.

In Fig. 4, the superposition of the two flow components during negative SIs for these two hypotheses is schematically illustrated as case A and case B, respectively. For comparison, case $\mathrm{C}$ with a constant round cell and symmetric flow vortices is also shown only for the left-hand panel. For the positive IMF-By case shown on the left, for example, the clockwise round cell (thick blue arrow on the top) dominates the polar ionospheric convection before SIs, and then a pair of transient SI convection vortices (light blue arrows, referred to as SI cell in the figure) are added to it during negative SIs. As a result, the higher-latitude portion of the dusk vortex and the pre-existing round cell flow gives an enhanced sunward flow in total, as marked by orange dashed circles in Fig. 4. The polarity of the round cell is reversed for the negative IMF-By case, leading to the enhancement of the higher-latitude sunward flow on the dawn side, as shown on the right-hand panel.

Note, however, that the flow perturbation deduced by the present analysis is the difference between the pre-SI convection and the total convection during SIs, not the flow pattern during SI itself. That is, the flow perturbation shown in Fig. 3 corresponds to a subtraction of the flow pattern on the top panels from that on the bottom panels in Fig. 4. Thus, one can expect naturally that the subtracted flow pattern should be a simple pair of symmetric vortices, if we assume that the round cell does not vary significantly between before and during SIs and the dawndusk symmetric vortices induced upon SIs, as illustrated by case $\mathrm{C}$. Conversely, the fact that the residual flow pattern shows a dawn-dusk asymmetric enhancement of the higher-latitude sunward flow implies that either assumption of the symmetry of vortices or the constant round cell could break for negative SIs, as illustrated by cases A and $\mathrm{B}$, respectively. In case A, the dusk (dawn) side SI cell becomes stronger, resulting in an enhancement of residual sunward flow on the dusk (dawn) side for positive (negative) IMF-By conditions. In case B, the round cell faster than its pre-SI level results in an enhanced sunward flow on dusk and reduced flow on dawn in the residual flow pattern for positive IMF-By cases, and vice versa for negative IMF-By. The dawn-dusk polarity of the enhancement of residual sunward flow is consistent with the negative SI results shown in Fig. 3. Therefore, both hypotheses could explain the observed asymmetry for negative SIs.

It is hard to prove or deny either of the two hypotheses solely by the present ionospheric observations. However, the SI-induced twin vortex-shaped convection cell reproduced by global MHD simulations for both positive SI (e.g., Samsonov and Sibeck 2013) and negative SI (e.g.,
Under positive IMF-By

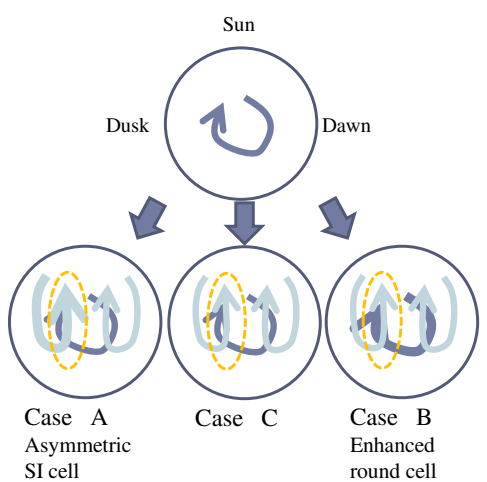

Under negative IMF-By

Round cell before SI

Round cell

+ SI cells

during SI -

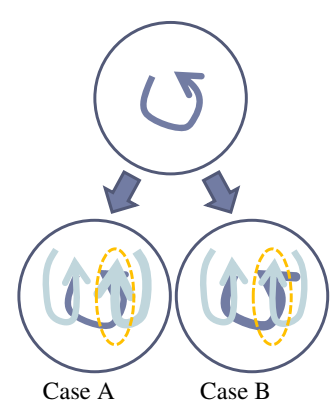

Fig. 4 Superposition of flows due to the pre-existing round cell and SI-induced twin vortices in the course of SI 
Fujita et al. 2004) seems to be fairly symmetric in the dawn-dusk direction. In particular, the latter result obtained by Fujita et al. (2004) resulted from a simulation under a finite IMF-By condition. Thus, these global MHD simulations suggest that case A may not be likely to occur, at least, from a theoretical point of view.

If case B was likely to cause the residual flow asymmetry during negative SIs, the pre-existing round cell should be enhanced during negative SIs than the pre-SI level. Since the round cell flow is driven by IMF-By, this case would imply that IMF-By becomes larger upon negative SIs. To test this hypothesis, we examine the IMF-By change across the jumps in solar wind dynamic pressure for the positive and negative IMF-By cases, again by separating the dataset into the positive and negative SIs. In Fig. 5, we plot the 30-min average IMFBy values after each SI onset against the 30-min averages of IMF-By just before SI onset for positive SI events (panel a) and negative SI events (panel b). Each point corresponds to each SI event. Black and red points represent those for positive and negative IMF-By events, respectively. As seen in Fig. 5, the results are clearly consistent with our expectation. Although the points are scattered much, the positive $P_{\mathrm{SW}}$ jumps are accompanied by increases and decreases of IMF-By intensity with roughly the same probability for positive SIs as shown by Fig. $5 \mathrm{a}$, while the negative $P_{\mathrm{SW}}$ jumps, causing negative SIs, bring along |IMF-By| increases with significantly better chance as evident from Fig. 5b. Therefore, the dawn-dusk asymmetry of flow perturbation associated with negative SIs can be attributed, at least, qualitatively to the enhancement of the pre-existing round convection cell as proposed in case B. On the contrary, the positive SI result, giving a similar residual flow pattern for both positive and negative IMF conditions, implies that case $C$ would be the case for positive SIs, because the constant round cell in flow intensity is expected on average from the result of Fig. 5a.

So far we do not have a clear answer for the reason why $P_{\mathrm{SW}}$ drops are preferentially accompanied by the IMF-By intensification. A possible speculation may be drawn from the study on the cause of negative SIs conducted by Takeuchi et al. (2002). Their statistics show that a negative SI often ( $50 \%)$ follows a preceding positive SI. This implies that a high-density structure of solar wind causes a positive SI first, and the subsequent low dynamic pressure wind gives rise to a negative SI. If the tailing boundary of the high-density wind is diamagnetic and the draping Parker-spiral IMF is compressed backward, it is likely to give the IMF-By field of the same polarity across the boundary with a larger By intensity behind. The similar enhancement of IMF-By could also be expected with the tangential discontinuity at the front boundary of a magnetic cloud, which actually was reported

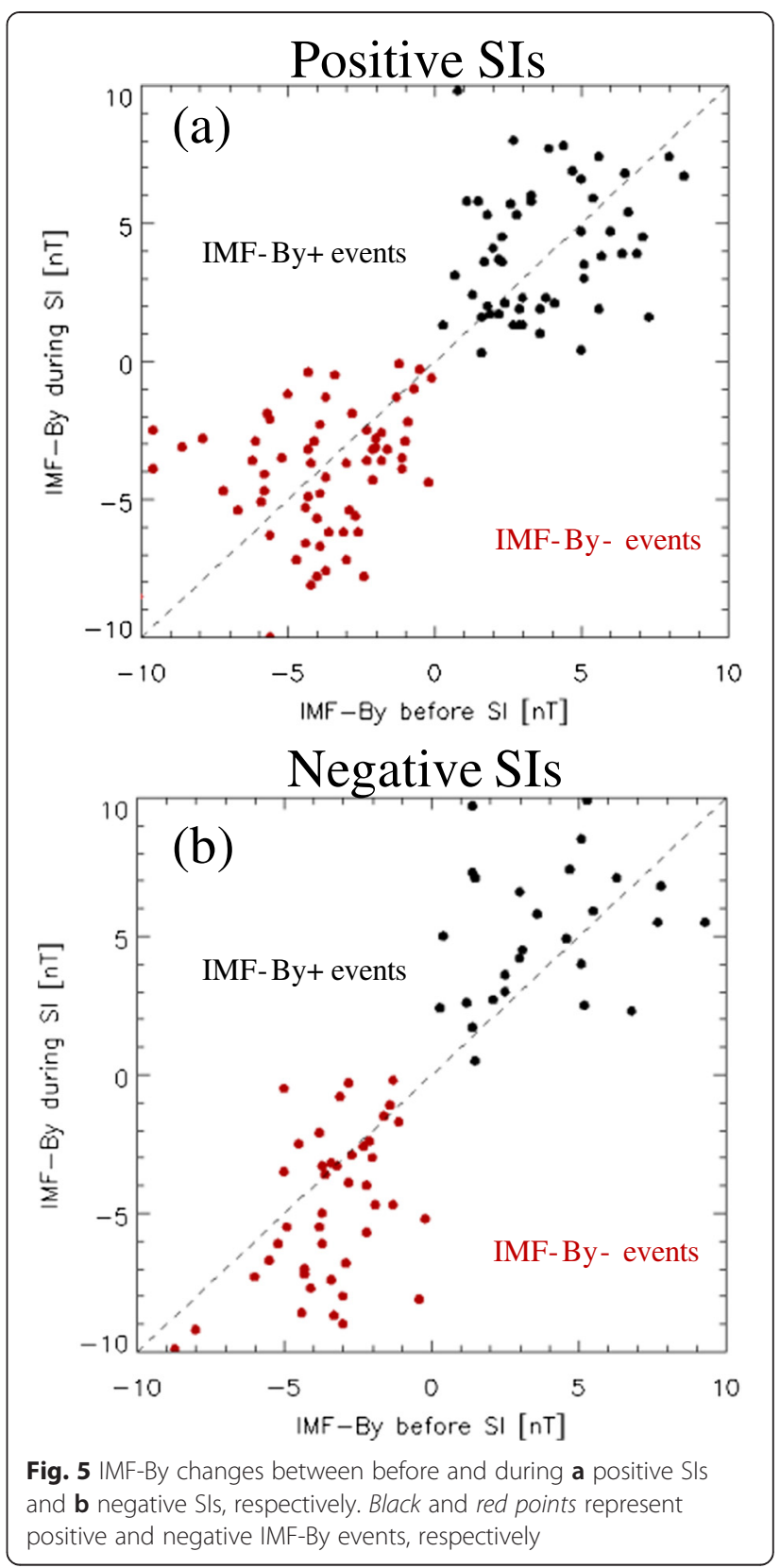

to cause a negative SI (Takeuchi et al. 2000). A detailed examination of the IMF structures across $P_{\mathrm{SW}}$ drops and its statistical study needs to be done to test this speculation, which is beyond the scope of the present study.

\section{Summary and conclusion}

The present study has analyzed the large dataset of SuperDARN observation to examine statistically ionospheric flow perturbation in association with SIs caused by sudden rises or drops of the solar wind dynamic pressure. It is revealed that negative SIs induce flow perturbation with a significant dawn-dusk asymmetry in flow intensity of a higher-latitude sunward flow, while such an asymmetry is 
not identified for positive SIs. A qualitative but likely interpretation is that the solar wind structures with abrupt drop of dynamic pressure are preferentially accompanied by an enhancement of IMF-By component. The enhanced IMF-By upon negative SIs strengthens flows of the preexisting round cell, resulting in the apparent dawn-dusk asymmetry of flow perturbation derived as the difference in flow pattern between pre-SI and SI intervals.

\section{Competing interests}

The authors declare that they have no competing interests.

\section{Authors' contributions}

$\mathrm{TH}$ performed the data processing and analysis described in the present article and gave the interpretation. AS, SF, and NN were heavily involved into discussions regarding the interpretations of the data and the statistical results presented here. All the authors have read and approved the final manuscript.

\section{Acknowledgements}

We would like to thank all the staffs involved in the SuperDARN project who made available the data to the present study. The FITACF CDF data of SuperDARN and the data analysis software used in the present study were distributed by ERG-Science Center (ERG-SC) operated by ISAS/JAXA and ISEE/Nagoya University. The ACE solar wind and magnetic field data were provided by the ACE Science data center as the OMNI data distributed by NASA/NSSDC. The SYM-H and AE indices were provided by World Data Center for Geomagnetism, Kyoto. A part of the work by T. H. was done at ERG-SC.

\section{Author details}

${ }^{1}$ Institute for Space-Earth Environmental Research, Nagoya University, Furo-cho, Chikusa, Nagoya, Aichi 464-8601, Japan. ${ }^{2}$ Research Institute for Sustainable Humanosphere, Kyoto University, Uji, Kyoto, Japan.

${ }^{3}$ Meteorological College, Kashiwa, Chiba, Japan.

\section{Received: 31 March 2015 Accepted: 19 November 2015}

Published online: 25 November 2015

\section{References}

Araki T (1977) Global structure of geomagnetic sudden commencements. Planet Space Sci 25:373-384. doi:10.1016/0032-0633(77)90053-8

Araki T (1994) A physical model of the geomagnetic sudden commencement. In: Engebretson MJ, Takahashi K, Scholer M (eds) Solar wind sources of magnetospheric ultra-low-frequency waves. American Geophysical Union, Washington

Araki T, Nagano H (1988) Geomagnetic response to sudden expansions of the magnetosphere. J Geophys Res 93:3983. doi:10.1029/JA093iA05p03983

Baker KB, Wing S (1989) A new magnetic coordinate system for conjugate studies at high latitudes. J Geophys Res 94:9139. doi:10.1029/JA094iA07p09139

Chao JK, Lepping RP (1974) A correlative study of ssc's, interplanetary shocks, and solar activity. doi:10.1029/JA079i013p01799

Coco I, Amata E, Marcucci MF, Ambrosino D, Villain J-P, Hanuise C (2008) The effects of an interplanetary shock on the high-latitude ionospheric convection during an IMF By-dominated period. Ann Geophys 26:2937-2951. doi:10.5194/angeo-26-2937-2008

Fujita S, Tanaka T, Kikuchi T, Tsunomura S (2004) A numerical simulation of a negative sudden impulse. Earth, Planets Space 56:463-472. doi:10.1186/BF03352499

Fujita S, Yamagishi H, Murata KT, Den M, Tanaka T (2012) A numerical simulation of a negative solar wind impulse: revisited. J Geophys Res 117:A09219. doi:10.1029/2012JA017526

Gillies DM, St.-Maurice J-P, McWilliams KA, Milan S (2012) Global-scale observations of ionospheric convection variation in response to sudden increases in the solar wind dynamic pressure. J Geophys Res 117:A04209. doi:10.1029/2011JA017255

Greenwald RA, Baker KB, Dudeney JR, Pinnock M, Jones TB, Thomas EC, Villain J-P, Cerisier J-C, Senior C, Hanuise C, Hunsucker RD, Sofko G, Koehler J, Nielsen E, Pellinen R, Walker ADM, Sato N, Yamagishi H (1995) DARN/SuperDARN. Space Sci Rev 71:761-796. doi:10.1007/BF00751350

Hori T, Shinbori A, Nishitani N, Kikuchi T, Fujita S, Nagatsuma T, Troshichev O, Yumoto K, Moiseyev A, Seki K (2012) Evolution of negative SI-induced ionospheric flows observed by SuperDARN King Salmon HF radar. J Geophys Res 117:A12223. doi:10.1029/2012JA018093

Huang CS, Yumoto K, Abe S, Sofko G (2008) Low-latitude ionospheric electric and magnetic field disturbances in response to solar wind pressure enhancements. J Geophys Res Space Phys 113:1-11. doi:10.1029/2007JA012940

Kane TA, Makarevich RA (2010) HF radar observations of the $F$ region ionospheric plasma response to storm sudden commencements. J Geophys Res Space Phys 115:1-13. doi:10.1029/2009JA014974

Lester M (2014) The Super Dual Auroral Radar Network (SuperDARN): an overview of its development and science. Adv Polar Sci 24:1-11. doi:10.3724/SP.J.1085.2013.00001

Liu JJ, Hu HQ, Han DS, Araki T, Hu ZJ, Zhang QH, Yang HG, Sato N, Yukimatu a S, Ebihara $Y$ (2011) Decrease of auroral intensity associated with reversal of plasma convection in response to an interplanetary shock as observed over Zhongshan station in Antarctica. J Geophys Res Space Phys 116:1-9. doi:10.1029/2010JA016156

Lyatsky W, Elphinstone RD, Pao Q, Cogger LL (1999) Field line resonance interference model for multiple auroral arc generation. J Geophys Res 104:263. doi:10.1029/1998JA900027

Makarevich RA, Dyson PL (2008) Dual HF radar study of the subauroral polarization stream. Ann Geophys 25:2579-2591. doi:10.5194/angeo-25-2579-2007

Nishida A, Jacobs JA (1962a) World-wide changes in the geomagnetic field. J Geophys Res 67:525-540. doi:10.1029/JZ067i002p00525

Nishida A, Jacobs JA (1962b) Equatorial enhancement of world-wide changes. J Geophys Res 67:4937-4940. doi:10.1029/JZ067i012p04937

Ruohoniemi JM, Greenwald RA (1996) Statistical patterns of high-latitude convection obtained from Goose Bay HF radar observations. J Geophys Res 101:21743. doi:10.1029/96JA01584

Samsonov AA, Sibeck DG (2013) Large-scale flow vortices following a magnetospheric sudden impulse. J Geophys Res Space Phys 118:3055-3064 doi:10.1002/jgra.50329

Shinbori A, Tsuji Y, Kikuchi T, Araki T, Ikeda A, Uozumi T, Baishev D, Shevtsov BM Nagatsuma T, Yumoto K (2012) Magnetic local time and latitude dependence of amplitude of the main impulse (MI) of geomagnetic sudden commencements and its seasonal variation. J Geophys Res 117:A08322. doi:10.1029/2012JA018006

Sundeen SR, Blanchard GT, Baker KB (2004) Reanalysis of criteria for identifying ground scatter. In: Abstract, 2004 SuperDARN Workshop (Saskatoon, Canada)

Takeuchi T, Araki T, Luehr H, Rasmussen O, Watermann J, Milling DK, Mann IR, Yumoto K, Shiokawa K, Nagai T (2000) Geomagnetic negative sudden impulse due to a magnetic cloud observed on May 13, 1995 doi:10.1029/2000JA900055

Takeuchi T, Araki T, Viljanen A, Watermann J (2002) Geomagnetic negative sudden impulses: interplanetary causes and polarization distribution. J Geophys Res Space Phys. doi:10.1029/2001JA900152

Tamao T (1964a) A hydromagnetic interpretation of geomagnetic SSC* . Rep Ionos Sp Res Jpn 18:16-31

Tamao T (1964b) The structure of three-dimensional hydromagnetic waves in a uniform cold plasma. J Geomagn Geoelectr 18:89-114

Tanaka T (1999) Configuration of the magnetosphere-ionosphere convection system under northward IMF conditions with nonzero IMF B y. J Geophys Res 104:14683. doi:10.1029/1999JA900077

Thorolfsson A, Cerisier J-C, Pinnock M (2001) Flow transients in the postnoon ionosphere: the role of solar wind dynamic pressure. 106:1887-1901. doi:10.1029/2000JA900078

Tsurutani BT, Gonzalez WD, Gonzalez ALC, Tang F, Arballo JK, Okada M (1995) Interplanetary origin of geomagnetic activity in the declining phase of the solar cycle. J Geophys Res 100:21717

Vichare G, Rawat R, Bhaskar A, Pathan BM (2014) Ionospheric current contribution to the main impulse of a negative sudden impulse. Earth, Planets Space 66:92. doi:10.1186/1880-5981-66-92

Vontrat-Reberac A, Cerisier J-C, Sato N, Lester M (2002) Noon ionospheric signatures of a sudden commencement following a solar wind pressure pulse. Ann Geophys 20:639-645. doi:10.5194/angeo-20-639-2002

Weimer DR (1995) Models of high-latitude electric potentials derived with a least error fit of spherical harmonic coefficients. J Geophys Res 100:19595. doi:10.1029/95JA01755

Wilken B, Goertz CK, Baker DN, Higbie PR, Fritz T a (1982) The SSC on July 29, 1977 and its propagation within the magnetosphere. J Geophys Res 87:5901. doi:10.1029/JA087iA08p05901 